PROCEEDINGS OF THE

AMERICAN MATHEMATICAL SOCIETY

Volume 136, Number 11, November 2008, Pages 4033-4043

S 0002-9939(08)09442-2

Article electronically published on June 5, 2008

\title{
LYAPUNOV EXPONENT ESTIMATES OF A CLASS OF HIGHER-ORDER STOCHASTIC ANDERSON MODELS
}

\author{
DAN TANG AND LIJUN BO \\ (Communicated by Edward C. Waymire)
}

\begin{abstract}
In this article, we propose a class of high-order stochastic partial differential equations (SPDEs) for spatial dimensions $d \leq 5$ which might be called high-order stochastic Anderson models. This class of the equations is perturbed by a space-time white noise when $d \leq 3$ and by a space-correlated Gaussian noise when $d=4,5$. The objectives of this article are to get some estimates on the Lyapunov exponent of the solutions and to study the convergence rates of the chaos expansions of the solutions for the models.
\end{abstract}

\section{INTRODUCTION}

In this section, we start at the following well-known Anderson model:

$$
\left\{\begin{array}{l}
\frac{\partial}{\partial t} u(t, x)=\Delta u(t, x)+u(t, x) \dot{W}(t, x), \text { in }[0, \infty) \times \mathbf{R}, \\
u(0, x)=u_{0}(x), \quad x \in \mathbf{R}
\end{array}\right.
$$

where $\dot{W}(t, x)$ is a space-time white noise on some probability space $(\Omega, \mathcal{F}, P)$ and satisfies $E[\dot{W}(t, x) \dot{W}(s, y)]=\delta(|t-s|) \delta(|x-y|)$ with $(t, x),(s, y) \in[0, \infty) \times \mathbf{R}$ formally. There have been extensive studies on this model (see e.g. [6, 7, 8, 9, 10, 11]). In those articles, the authors proved the existence and uniqueness of the solution and studied at greater length the asymptotic behaviors of the solution to (1.1). The key role in those approaches is the Itô-Wiener chaos expansion. On the other hand, since the mild solution of (1.1) in Walsh's [12] sense is function-valued if and only if $d=1$. For higher dimensions $d \geq 2$, to get a function-valued solution to (1.1), one has to introduce the stochastic integral of Skorohod type, which might be found in more detail in [6, 10, 11].

In this article, we are interested in a class of higher order stochastic partial differential equations (SPDEs), which goes back to the well-known (deterministic) Cahn-Hilliard equations in material sciences. By the deterministic model, CardonWeber [3] (see also Bo and Wang 1]) established a class of higher order SPDEs (called Stochastic Cahn-Hilliard equations). Here we focus a concrete linear highorder SPDE, which might be regarded as a higher-order stochastic Anderson model.

Received by the editors October 18, 2007.

2000 Mathematics Subject Classification. Primary 60H15, 34A34, 49N60.

Key words and phrases. High-order SPDEs, stochastic Anderson models, Lyapunov exponent, convergence rates.

This work was supported by the LPMC at Nankai University and the NSF of China (No. 10471003).

(C)2008 American Mathematical Society Reverts to public domain 28 years from publication 
This equation is described by the following:

$$
\left\{\begin{array}{l}
\left(\frac{\partial}{\partial t}+\Delta^{2}\right) u(t, x)=u(t, x) \dot{W}(t, x), \text { in }[0, \infty) \times \mathbf{D} \\
u(0, x)=u_{0}(x), x \in \mathbf{D}, \\
\frac{\partial u(t, x)}{\partial n}=\frac{\partial \Delta u(t, x)}{\partial n}=0, \text { on }[0, \infty) \times \partial \mathbf{D}
\end{array}\right.
$$

where the domain $\mathbf{D}=[0, \pi]^{d}$ with $d \leq 3$. For Equation (1.2), we say $u$ is a solution of (1.2) in a weak sense as in [12] if $u$ satisfies that

$$
u(t, x)=\int_{\mathbf{D}} G(t, x, y) u_{0}(y) d y+\int_{0}^{t} \int_{\mathbf{D}} G(t-s, x, y) u(s, y) W(d y, d s),
$$

where $G(t, x, y):(t, x, y) \in[0, \infty) \times \mathbf{D}^{2} \rightarrow \mathbf{R}$ is the Green kernel of the operator $\frac{\partial}{\partial t}+\Delta^{2}$ with a homogeneous Neumann boundary condition as in (1.2). We notice that the existence and uniqueness have been proved for the function-valued mild solution of (1.2) with $d \leq 3$ in Bo et al. [2].

On the other hand, we shall consider Equation (1.2) for $d=4,5$. In order to get a function-valued solution for (1.2), we have to replace the space-time white noise in (1.2) by a space-correlated Gaussian noise. That is, for $d=4,5$, we consider such an SPDE as follows:

$$
\left\{\begin{array}{l}
\left(\frac{\partial}{\partial t}+\Delta^{2}\right) u(t, x)=u(t, x) \dot{F}(t, x), \text { in }[0, \infty) \times \mathbf{D}, \\
u(0, x)=u_{0}(x), x \in \mathbf{D}, \\
\frac{\partial u(t, x)}{\partial n}=\frac{\partial \Delta u(t, x)}{\partial n}=0, \text { on }[0, \infty) \times \partial \mathbf{D},
\end{array}\right.
$$

where the random field $F:=\left(F(\varphi), \varphi \in \mathcal{D}\left(\mathbf{R}_{+} \times \mathbf{D}\right)\right)$ is an $L^{2}(\Omega)$-valued Gaussian field. For $\varphi, \psi \in \mathcal{D}\left(\mathbf{R}_{+} \times \mathbf{D}\right)$, the covariance of $F(\varphi)$ and $F(\psi)$ is given by

$$
J(\varphi, \psi):=E[F(\varphi) F(\psi)]=\int_{0}^{\infty} \int_{\mathbf{D}^{2}} \varphi(t, y) f(y-z) \psi(t, z) d y d z d t,
$$

where $f:(\mathbf{D}-\mathbf{D})^{*} \rightarrow[0,+\infty)$ is a continuous kernel function with $(\mathbf{D}-\mathbf{D})^{*}:=$ $\{y-z ; y, z \in \mathbf{D}, y \neq z\}$. The bilinear form $J$ defined by (1.5) is non-negative definite if and only if $f$ is the Fourier transform of a non-negative tempered distribution $\mu$ on $\mathbf{D}$ (see [5]). Thus $F$ defines a martingale measure on which the stochastic integrals are well defined in Walsh's [12 sense. Throughout the paper, we take $f(r)=|r|^{-\alpha}$ for some $\alpha \in\left(0, d-\frac{4}{3}\right)$. It follows from the standard argument in [4] that Equation (1.4) admits a unique function-valued solution for $d=4,5$.

Our central objective is to estimate the Lyapunov exponents in $L^{2}(\Omega)$ for the solutions of Equations (1.2) and (1.4). Besides this, the convergence rate of the solutions by their finite chaos expansion terms will also be given in the latter.

\section{LyapunOV EXPONENT ESTIMATES FOR the SOlution OF (1.2)}

At first we have to address the existence and the uniqueness of the solution to Equation (1.2) (the proof refers to Bo et al. 2]).

Proposition 2.1. Let $\mathbf{D}=[0, \pi]^{d}$ with $d \leq 3$. Consider the following SPDE:

$$
\left\{\begin{array}{l}
\left(\frac{\partial}{\partial t}+\Delta^{2}\right) u=b(u)+a(u) \dot{W}, \text { in }[0, \infty) \times \mathbf{D}, \\
u(0, x)=u_{0}(x), \quad x \in \mathbf{D}, \\
\frac{\partial u}{\partial n}=\frac{\partial \Delta u}{\partial n}=0, \text { on }[0, \infty) \times \partial \mathbf{D} .
\end{array}\right.
$$

Assume that $b(\cdot), a(\cdot)$ are all Lipschitzian on $\mathbf{R}$ satisfying linear growth conditions and the initial $u_{0} \in L^{q}(\mathbf{D})$ with $2 \leq q \leq \infty$. 
(1) If $q=\infty$, then for every $T>0,(2.1)$ admits a unique solution $(u(t, x))_{(t, x) \in[0, T] \times \mathbf{D}}$ such that for $p \in[2, \infty)$,

$$
\sup _{(t, x) \in[0, T] \times \mathbf{D}} E\left[|u(t, x)|^{p}\right]<\infty .
$$

(2) If $2 \leq q \leq p<\infty$ and $d \in\{1,2\}$, then for every $T>0$, (2.1) admits a unique solution $(u(t, x))_{(t, x) \in[0, T] \times \mathbf{D}}$ such that

$$
\sup _{t \in[0, T]} E\left[\|u(t, \cdot)\|_{q}^{p}\right]<\infty .
$$

(3) If $2 \leq q \leq p<\infty, \frac{1}{q}-\frac{1}{p}<\frac{1}{6}$ and $d=3$, then for every $T>0$, (2.1) admits a unique solution $(u(t, x))_{(t, x) \in[0, T] \times \mathbf{D}}$ such that

$$
\sup _{t \in[0, T]} E\left[\|u(t, \cdot)\|_{q}^{p}\right]<\infty
$$

In our model (1.2), set $a(u)=u$ and $b(u) \equiv 0$ in (2.1). Then by Proposition 2.1, Equation (1.2) possesses a unique solution $(u(t, x))_{(t, x) \in[0, T] \times \mathbf{D}}$ for every $T>0$. For this solution, we shall have a Lyapunov exponent uniform estimate, which is explicitly stated as follows:

Theorem 2.1. Let $d \leq 3$ and the initial $u_{0} \in L^{\infty}(\mathbf{D})$. Then for all small $\varepsilon>0$,

$$
\limsup _{t \rightarrow \infty} \frac{\log \left\{\sup _{x \in \mathbf{D}} E\left[|u(t, x)|^{2}\right]\right\}}{t^{1+\varepsilon}} \leq h(\varepsilon, d),
$$

where the upper bound $h(\varepsilon, d)$ is given by

$$
h(\varepsilon, d)=\frac{1-d / 4}{1+\varepsilon}\left[\frac{\varepsilon+d / 4}{\varepsilon(1-d / 4)}\right]^{\frac{\varepsilon+d / 4}{1-d / 4}} .
$$

To prove Theorem 2.1, we need the following estimates on the Green function $G$ corresponding to the operator $\frac{\partial}{\partial t}+\Delta^{2}$ with a homogeneous Neumann boundary condition as in (1.2).

Lemma 2.1 ([3]). For $(t, x, y) \in(0, \infty) \times \mathbf{D}^{2}$, there exist positive constants $k_{1}:=$ $k_{1}(d), k_{2}:=k_{2}(d)$ such that

$$
|G(t, x, y)| \leq \frac{k_{1}}{t^{\frac{d}{4}}} \exp \left(-k_{1} \frac{|x-y|^{\frac{4}{3}}}{t^{\frac{1}{3}}}\right)
$$

and

$$
\int_{\mathbf{R}^{d}} \exp \left(-\frac{|x|^{\frac{4}{3}}}{t^{\frac{1}{3}}}\right) d x=k_{2} t^{\frac{d}{4}}
$$

Now we are in a position to prove Theorem 2.1.

Proof of Theorem 2.1. We first introduce the series $\left\{H_{i}(t, x), i \geq 0\right\}$ and $\left\{I_{i}(t, x)\right.$, $i \geq 0\}$. For $i=1,2, \cdots$, and $(t, x) \in[0, \infty) \times \mathbf{D}$, define

$$
\begin{aligned}
H_{0}(t, x)= & \int_{\mathbf{D}} G(t, x, y) u_{0}(y) d y \\
H_{i}(t, x)= & \int_{0}^{t} \int_{0}^{s_{1}} \cdots \int_{0}^{s_{i-1}} \int_{\mathbf{D}^{i+1}} G\left(t-s_{1}, x, y_{1}\right) \times \cdots \times G\left(s_{i-1}-s_{i}, y_{i-1}, y_{i}\right) \\
& \times G\left(s_{i}, y_{i}, y_{i+1}\right) u_{0}\left(y_{i+1}\right) d y_{i+1} W\left(d s_{i}, d y_{i}\right) \cdots W\left(d s_{1}, d y_{1}\right)
\end{aligned}
$$


and

$$
\begin{aligned}
I_{0}(t, x)= & {\left[\int_{\mathbf{D}} G(t, x, y) u_{0}(y) d y\right]^{2}, } \\
I_{i}(t, x)= & \int_{0}^{t} \int_{0}^{s_{1}} \cdots \int_{0}^{s_{i-1}} \int_{\mathbf{D}^{i}} G^{2}\left(t-s_{1}, x, y_{1}\right) \times \cdots \times G^{2}\left(s_{i-1}-s_{i}, y_{i-1}, y_{i}\right) \\
& \times\left[\int_{\mathbf{D}} G\left(s_{i}, y_{i}, y_{i+1}\right) u_{0}\left(y_{i+1}\right) d y_{i+1}\right]^{2} d y_{i} d s_{i} \cdots d y_{1} d s_{1} .
\end{aligned}
$$

Next we are going to estimate $I_{i}(t, x)$ with $i=1,2, \cdots$. Note that $u_{0} \in L^{\infty}(\mathbf{D})$. Then by Lemma 2.1,

$$
\begin{aligned}
\int_{\mathbf{D}} G\left(s_{i}, y_{i}, y_{i+1}\right) u_{0}\left(y_{i+1}\right) d y_{i+1} & \leq\left\|u_{0}\right\|_{\infty}\left[\int_{\mathbf{D}}\left|G\left(s_{i}, y_{i}, y_{i+1}\right)\right| d y_{i+1}\right] \\
& =\text { const. }
\end{aligned}
$$

From Lemma 2.1, it follows that for all $(t, x) \in[0, \infty) \times \mathbf{D}$,

$$
\begin{aligned}
& I_{i}(t, x) \\
\leq & \text { const. } \int_{0}^{t} \int_{0}^{s_{1}} \cdots \int_{0}^{s_{i-1}} \frac{1}{\left|t-s_{1}\right|^{\frac{d}{4}}} \times \cdots \times \frac{1}{\left|s_{i-2}-s_{i-1}\right|^{\frac{d}{4}}} \times \frac{1}{\left|s_{i-1}-s_{i}\right|^{\frac{d}{4}}} d s_{1} \cdots d s_{i} \\
= & \text { const. } \int_{0}^{t} \int_{0}^{s_{1}} \cdots \int_{0}^{s_{i-2}} \frac{1}{\left|t-s_{1}\right|^{\frac{d}{4}}} \times \cdots \times \frac{1}{\left|s_{i-2}-s_{i-1}\right|^{\frac{d}{4}}} \times s_{i-1}^{1-\frac{d}{4}} d s_{1} \cdots d s_{i-1} .
\end{aligned}
$$

A routine calculation shows that, for $\alpha>0$ and $j=1,2, \cdots$,

$$
\begin{aligned}
\int_{0}^{s_{j}} \frac{s_{j+1}^{\alpha}}{\left|s_{j}-s_{j+1}\right|^{\frac{d}{4}}} d s_{j+1} & =\int_{0}^{s_{j}} \frac{s_{j+1}^{\alpha} s_{j}^{-\frac{d}{4}}}{\left|1-\frac{s_{j+1}}{s_{j}}\right|^{\frac{d}{4}}} d s_{j+1} \\
& =s_{j}^{\alpha+\left(1-\frac{d}{4}\right)} \int_{0}^{1} a^{\alpha}(1-a)^{-\frac{d}{4}} d a .
\end{aligned}
$$

Thus by $(2.6)$, for all $(t, x) \in[0, \infty) \times \mathbf{D}$,

$$
I_{i}(t, x) \leq \text { const. } t^{i\left(1-\frac{d}{4}\right)} \prod_{k=1}^{i-1} \int_{0}^{1} a^{k\left(1-\frac{d}{4}\right)}(1-a)^{-\frac{d}{4}} d a,
$$

where we set $\prod_{k=1}^{0} \equiv 1$ by the usual convention. Note that for $k=1,2, \cdots$, and $1<p<\frac{4}{d}$ with $\frac{1}{q}+\frac{1}{p}=1$,

$$
\begin{aligned}
\int_{0}^{1} a^{k\left(1-\frac{d}{4}\right)}(1-a)^{-\frac{d}{4}} d a & \leq\left[\int_{0}^{1}(1-a)^{-\frac{d p}{4}} d a\right]^{\frac{1}{p}} \times\left[\int_{0}^{1}(1-a)^{\left(1-\frac{d}{4}\right) k q} d a\right]^{\frac{1}{q}} \\
& =\left[\frac{1}{1-\frac{d p}{4}}\right]^{\frac{1}{p}}\left[\frac{1}{1+\left(1-\frac{d}{4}\right) k q}\right]^{\frac{1}{q}}
\end{aligned}
$$


Therefore by (2.7),

$$
\begin{aligned}
I_{i}(t, x) & \leq \text { const. } t^{i\left(1-\frac{d}{4}\right)} \prod_{k=1}^{i-1} \int_{0}^{1} a^{k\left(1-\frac{d}{4}\right)}(1-a)^{-\frac{d}{4}} d a \\
& \leq \text { const. }\left[\frac{1}{1-\frac{d p}{4}}\right]^{\frac{i-1}{p}} \frac{t^{i\left(1-\frac{d}{4}\right)}}{\left[\left(1-\frac{d}{4}\right) q\right]^{\frac{i-1}{q}}[(i-1) !]^{\frac{1}{q}}} .
\end{aligned}
$$

Let

$$
u(t, x)=\sum_{i=0}^{\infty} H_{i}(t, x)
$$

and so

$$
E\left[|u(t, x)|^{2}\right]=\sum_{i=0}^{\infty} I_{i}(t, x)
$$

From (2.9) and the Hölder inequality, it follows that there exist constants $c_{1}, c_{2}>0$ such that

$$
\begin{aligned}
E\left[|u(t, x)|^{2}\right] \leq & c_{1}+c_{2} \sum_{i=1}^{\infty} t^{i\left(1-\frac{d}{4}\right)}\left[\frac{1}{1-\frac{d p}{4}}\right]^{\frac{i-1}{p}} \times \frac{1}{\left[\left(1-\frac{d}{4}\right) q\right]^{\frac{i-1}{q}}[(i-1) !]^{\frac{1}{q}}} \\
\leq & \left.c_{1}+c_{2} t^{1-\frac{d}{4}} \sum_{i=1}^{\infty}\left[\frac{t^{1-\frac{d}{4}}}{\left(1-\frac{d p}{4}\right)^{\frac{1}{p}}} \frac{1}{\left[q\left(1-\frac{d}{4}\right)\right]^{\frac{1}{q}}}\right]^{\frac{1}{q}} \times\left[\frac{1}{(i-1) !}\right]^{\frac{(i-1) p}{q}}\right\}^{\frac{1}{p}} \\
\leq & \left.c_{1}+c_{2} t^{1-\frac{d}{4}}\left\{\sum_{i=1}^{\infty}\left[\frac{1}{q\left(1-\frac{d}{4}\right)}\right]^{\frac{1}{q}}\right\}^{\frac{1}{q}}\right]^{-q / p}\left[\frac{1}{(i-1) !}\right]
\end{aligned}
$$

On the other hand, since $d \leq 3,1<p<4 / d, 1 / p+1 / q=1$, and so $q[1-d / 4]>1$. Then

$$
c_{3}(d)=:\left\{\sum_{i=1}^{\infty}\left[\frac{1}{q\left(1-\frac{d}{4}\right)}\right]^{\frac{(i-1) p}{q}}\right\}^{\frac{1}{p}}<\infty .
$$

Hence by $(2.12)$, we obtain for all $(t, x) \in[0, \infty) \times \mathbf{D}$,

$$
E\left[|u(t, x)|^{2}\right] \leq c_{1}+c_{2} c_{3}(d) t^{1-\frac{d}{4}} \exp \left(\frac{t^{\left(1-\frac{d}{4}\right) q}}{q\left(1-\frac{d}{4} p\right)^{\frac{q}{p}}}\right) .
$$

Recall the definitions of $H_{i}(t, x)$ with $i=1,2, \cdots$. By (2.10)-(2.13), we get that $u(t, x)$ defined by $(2.10)$ is a series solution of Equation (1.2). Moreover the uniqueness argument shows that it is the unique solution. In the following, we are going 
to get an estimate of the Lyapunov exponent for the solution. For any fixed $\epsilon>0$, set

$$
p=\frac{1+\varepsilon}{\frac{d}{4}+\varepsilon}<\frac{4}{d}, \quad q=\frac{1+\varepsilon}{1-\frac{d}{4}} .
$$

Then we apply the estimate (2.13) to conclude that

$$
\limsup _{t \rightarrow \infty} \frac{\log \left\{\sup _{x \in \mathbf{D}} E\left[|u(t, x)|^{2}\right]\right\}}{t^{1+\varepsilon}} \leq \frac{1-\frac{d}{4}}{1+\varepsilon}\left[\frac{\varepsilon+\frac{d}{4}}{\varepsilon\left(1-\frac{d}{4}\right)}\right]^{\frac{\varepsilon+\frac{d}{4}}{1-\frac{d}{4}}} .
$$

Then the desired result follows from the definition of $h(\varepsilon, d)$.

The next assertion gives the convergence estimate for the solution to (1.2) by its first $N$-finite chaos terms of (2.10).

Proposition 2.2. Let $d \leq 3$ and $N \in \mathbf{N}$. Define the first $N$-finite chaos terms of (2.8) by

$$
u_{N}(t, x)=\sum_{i=0}^{N} H_{i}(t, x) .
$$

For $q>\frac{4}{4-d}$ and $\alpha:=T^{\left(1-\frac{d}{4}\right) q}\left(1-\frac{d}{4} p\right)^{-q / p}$ with $T>0$, define

$$
b(d, N):=\left[\frac{\alpha}{q\left(1-\frac{d}{4}\right)}\right]^{\frac{N}{q}} \times \frac{1}{(N !)^{\frac{1}{q}}} \times\left[\frac{N}{N-\alpha}\right]^{\frac{1}{q}}
$$

and $\lim _{N \rightarrow \infty} b(d, N)=0$. Then there exists a constant $c:=c(d)>0$ such that

$$
E\left[\left|u(t, x)-u_{N}(t, x)\right|^{2}\right] \leq c b(d, N),
$$

for all $(t, x) \in[0, T] \times \mathbf{D}$ and $N>\alpha$.

Proof. By (2.10)-(2.11), we obtain for all $(t, x) \in[0, T] \times \mathbf{D}$,

$$
\begin{aligned}
& E\left[\left|u(t, x)-u_{N}(t, x)\right|^{2}\right] \\
& \quad=\sum_{i=N+1}^{\infty} I_{i}(t, x) \\
& \quad \leq \text { const. } \sum_{i=N+1}^{\infty} t^{i\left(1-\frac{d}{4}\right)}\left[\frac{1}{1-\frac{d p}{4}}\right]^{\frac{i-1}{p}} \times \frac{1}{\left[\left(1-\frac{d}{4}\right) q\right]^{\frac{i-1}{q}}[(i-1) !]^{\frac{1}{q}}} \\
& \quad \leq \text { const. } T^{1-\frac{d}{4}} \sum_{i=N+1}^{\infty}\left[T^{1-\frac{d}{4}} \frac{1}{\left(1-\frac{d p}{4}\right)^{\frac{1}{p}}} \frac{1}{\left[q\left(1-\frac{d}{4}\right)\right]^{\frac{1}{q}}}\right]^{i-1} \times\left[\frac{1}{(i-1) !}\right]^{\frac{1}{q}} \\
& \quad \leq \text { const. } A_{1}(N) \times A_{2}(N),
\end{aligned}
$$

with

$$
\begin{aligned}
A_{1}(N) & :=\left\{\sum_{i=N+1}^{\infty}\left[\frac{1}{q\left(1-\frac{d}{4}\right)}\right]^{\frac{(i-1) p}{q}}\right\}^{\frac{1}{p}} \\
& =\left[\frac{1}{q\left(1-\frac{d}{4}\right)}\right]^{\frac{N}{q}}\left\{\frac{1}{1-\left[\frac{1}{q\left(1-\frac{d}{4}\right)}\right]^{\frac{p}{q}}}\right\}^{\frac{1}{p}}
\end{aligned}
$$


and

$$
A_{2}(N):=\left\{\sum_{i=N+1}^{\infty}\left[T^{\left(1-\frac{d}{4}\right) q}\left(1-\frac{d}{4} p\right)^{-q / p}\right]^{i-1} \times\left[\frac{1}{(i-1) !}\right]\right\}^{\frac{1}{q}} .
$$

We take $N$ such that $0<\alpha:=T^{\left(1-\frac{d}{4}\right) q}\left(1-\frac{d}{4} p\right)^{-q / p}<N$. Therefore,

$$
\begin{aligned}
\sum_{i=N+1}^{\infty} \frac{\alpha^{i-1}}{(i-1) !} & =\sum_{i=0}^{\infty} \frac{\alpha^{N+i}}{(N+i) !} \\
& \leq \frac{\alpha^{N}}{N !} \sum_{i=0}^{\infty} \frac{\alpha^{i}}{N^{i}} \\
& =\frac{\alpha^{N}}{N !} \times \frac{N}{N-\alpha} .
\end{aligned}
$$

Then for $N>\alpha$,

$$
A_{2}(N) \leq \frac{\alpha^{\frac{N}{q}}}{(N !)^{\frac{1}{q}}}\left[\frac{N}{N-\alpha}\right]^{\frac{1}{q}}
$$

Then the conclusion of the proposition follows from (2.15).

\section{LyAPUNOV EXPONENT ESTimates FOR $d=4,5$}

In this section, we consider Equation (1.4) for $d=4,5$. As in the weak sense of [12, (1.4) is equivalent to the following mild form:

$$
u(t, x)=\int_{\mathbf{D}} G(t, x, y) u_{0}(y) d y+\int_{0}^{t} \int_{\mathbf{D}} G(t-s, x, y) u(s, y) F(d y, d s) .
$$

The existence and uniqueness of (3.1) have been established in [4 in the case of $f(r)=r^{-\alpha}$ (Riesz potential) for $\alpha \in\left(0, d-\frac{4}{3}\right)$, where $f$ is the spatial correlative kernel of $F$ as in (1.5). For $i=1,2, \cdots$ and $(t, x) \in[0, \infty) \times \mathbf{D}$, define

$$
\begin{aligned}
\widetilde{H}_{0}(t, x)= & \int_{\mathbf{D}} G(t, x, y) u_{0}(y) d y \\
\widetilde{H}_{i}(t, x)= & \int_{0}^{t} \int_{0}^{s_{1}} \cdots \int_{0}^{s_{i-1}} \int_{\mathbf{D}^{i+1}} G\left(t-s_{1}, x, y_{1}\right) \times \cdots \times G\left(s_{i-1}-s_{i}, y_{i-1}, y_{i}\right) \\
& \times G\left(s_{i}, y_{i}, y_{i+1}\right) u_{0}\left(y_{i+1}\right) d y_{i+1} F\left(d y_{i}, d s_{i}\right) \cdots F\left(d y_{1}, d s_{1}\right)
\end{aligned}
$$

and

$$
\begin{aligned}
\widetilde{I}_{0}(t, x)= & {\left[\int_{\mathbf{D}} G(t, x, y) u_{0}(y) d y\right]^{2}, } \\
\widetilde{I}_{i}(t, x)= & \int_{0}^{t} \int_{0}^{s_{1}} \cdots \int_{0}^{s_{i-1}} \int_{\mathbf{D}^{2 i}} G\left(t-s_{1}, x, y_{1}\right) f\left(y_{1}-z_{1}\right) G\left(t-s_{1}, x, z_{1}\right) \\
& \times \cdots \times G\left(s_{i-1}-s_{i}, y_{i-1}, y_{i}\right) f\left(y_{i}-z_{i}\right) \\
& \times G\left(s_{i-1}-s_{i}, y_{i-1}, z_{i}\right) d y_{i} d z_{i} d s_{i} \cdots d y_{1} d z_{1} d s_{1} \\
& \times\left[\int_{\mathbf{D}^{2}} G\left(s_{i}, y_{i}, y\right) u_{0}(y) f(y-z) G\left(s_{i}, y_{i}, z\right) u_{0}(z) d y d z\right] .
\end{aligned}
$$

Similarly as in Section 2, we shall have 
Theorem 3.1. Let $d=4,5$. Assume that the initial $u_{0} \in L^{\infty}(\mathbf{D})$. Then $\widetilde{I}_{i}(t, x)$ is well defined, for all $i \in \mathbf{N}$ and $(t, x) \in[0, \infty) \times \mathbf{D}$. Further, it holds that

$$
\limsup _{t \rightarrow \infty} \frac{\log \left\{\sup _{x \in \mathbf{D}} E\left[|u(t, x)|^{2}\right]\right\}}{t^{1+\left(\frac{4}{3}-\frac{d}{4}\right)}} \leq \frac{1}{\frac{7}{3}-\frac{d}{4}} .
$$

For proving Theorem 3.1, we need the following estimate.

Lemma 3.1. Let $d=4,5$ and $\alpha \in\left(0, d-\frac{4}{3}\right)$. Define

$$
I(t, x)=\int_{0}^{t} \int_{\mathbf{D}^{2}}|G(t-s, x, y)||y-z|^{-\alpha}|G(t-s, x, z)| d y d z d s .
$$

Then there exists a constant $c>0$ such that

$$
I(t, x) \leq c t^{\frac{4}{3}-\frac{d}{4}}, \quad \forall(t, x) \in[0, \infty) \times \mathbf{D} .
$$

Proof. Note that for $\beta \geq 1$ and $x, y, z \in \mathbf{R}$,

$$
|y-z|^{\beta} \leq 2^{\beta-1}\left[|x-y|^{\beta}+|x-z|^{\beta}\right] .
$$

Then by Lemma 2.1, for $\lambda \in(0,1)$,

$$
\begin{aligned}
& I(t, x) \\
\leq & k_{1}^{2} \int_{0}^{t} \int_{\mathbf{D}^{2}}(t-s)^{-\frac{d}{2}} \exp \left(-k_{1} \frac{|x-y|^{\frac{4}{3}}}{(t-s)^{\frac{1}{3}}}\right)|y-z|^{-\alpha} \exp \left(-k_{1} \frac{|x-z|^{\frac{4}{3}}}{(t-s)^{\frac{1}{3}}}\right) d y d z d s \\
= & k_{1}^{2} \int_{0}^{t} \int_{\mathbf{D}^{2}} s^{-\frac{d}{2}} \exp \left(-k_{1}(1-\lambda) \frac{|x-y|^{\frac{4}{3}}}{s^{\frac{1}{3}}}\right) \exp \left(-k_{1} \lambda \frac{|x-y|^{\frac{4}{3}}}{s^{\frac{1}{3}}}\right) \\
& \times|y-z|^{-\alpha} \exp \left(-k_{1} \lambda \frac{|x-z|^{\frac{4}{3}}}{s^{\frac{1}{3}}}\right) d y d z d s \\
\leq & k_{1}^{2} \int_{0}^{t} \int_{\mathbf{D}^{2}} s^{-\frac{d}{2}} \exp \left(-k_{1}(1-\lambda) \frac{|x-y|^{\frac{4}{3}}}{s^{\frac{1}{3}}}\right) \\
& \times|y-z|^{-\alpha} \exp \left(-k_{1} \lambda 2^{-\frac{1}{3}} \frac{|y-z|^{\frac{4}{3}}}{s^{\frac{1}{3}}}\right) d y d z d s \\
\leq & k_{1}^{2} \int_{0}^{t} s^{-\frac{d}{2}} \int_{\mathbf{D}} \exp \left(-\bar{c} \frac{|x-y|^{\frac{4}{3}}}{s^{\frac{1}{3}}}\right) d y \int_{\mathbf{D}}|y-z|^{-\alpha} \exp \left(-\bar{c} \frac{|y-z|^{\frac{4}{3}}}{s^{\frac{1}{3}}}\right) d z d s .
\end{aligned}
$$

Let $l=\left|s^{\frac{1}{4}}(x-y)\right|, v=y-z$. Then

$$
\begin{aligned}
I(t, x) & \leq \text { const. } \quad \int_{0}^{t} s^{\frac{d}{4}-\frac{d}{2}} \int_{0}^{\infty} \exp \left(-\bar{c} l^{\frac{4}{3}}\right) l^{d-1} d l \int_{B_{d}(0, R)} \exp \left(-\bar{c} \frac{|v|^{\frac{4}{3}}}{s^{\frac{1}{3}}}\right)|v|^{-\alpha} d v d s \\
& \leq \text { const. } \quad \int_{0}^{t} s^{-\frac{d}{4}} \int_{B_{d}(0, R)} \exp \left(-\bar{c} \frac{|v|^{\frac{4}{3}}}{s^{\frac{1}{3}}}\right)|v|^{-\alpha} d v d s
\end{aligned}
$$


where $B_{d}(0, R)$ is the ball centered at 0 with radius $R>2 \pi$. For $v \neq 0$ fixed, change variable by letting $r=|v|^{\frac{4}{3}} s^{-\frac{1}{3}}$. Then

$$
I(t, x) \leq \text { const. } \quad \int_{B_{d}(0, R)}|v|^{4-d-\alpha} d v \int_{|v|^{\frac{4}{3} t^{-\frac{1}{3}}}}^{\infty} r^{-4+\frac{3}{4} d} \exp (-\bar{c} r) d r .
$$

Note that for $d=4,5, \frac{3}{4} d-4<0$. Hence

$$
\begin{aligned}
I(t, x) & \leq \text { const. } \int_{B_{d}(0, R)}|v|^{4-d-\alpha}\left(|v|^{\frac{4}{3}} t^{-\frac{1}{3}}\right)^{-4+\frac{3}{4} d} d v \\
& =\text { const. } t^{\frac{4}{3}-\frac{d}{4}} \int_{B_{d}(0, R)}|v|^{-\frac{4}{3}-\alpha} d v \\
& \leq \text { const. } t^{\frac{4}{3}-\frac{d}{4}}
\end{aligned}
$$

for any $\alpha \in\left(0, d-\frac{4}{3}\right)$. Thus the proof of the lemma is completed.

In what follows, we proceed to the proof of Theorem 3.1.

Proof of Theorem 3.1. Applying the same iterative scheme as in Theorem 2.1, we have

$$
u(t, x)=\sum_{i=0}^{\infty} \widetilde{H}_{i}(t, x)
$$

and

$$
E\left[|u(t, x)|^{2}\right]=\sum_{i=0}^{\infty} \widetilde{I}_{i}(t, x) .
$$

Next we estimate the term $\widetilde{I}_{i}$ defined by (3.3) for $i \geq 1$. By Lemma 3.1, for all $(t, x) \in[0, \infty) \times \mathbf{D}$,

$$
\begin{aligned}
\widetilde{I}_{i}(t, x) \leq & \text { const. } \quad \int_{0}^{t} \int_{0}^{s_{1}} \cdots \int_{0}^{s_{i-1}}\left|s_{i-1}-s_{i}\right|^{\frac{4}{3}-\frac{d}{4}} \times\left|s_{i-2}-s_{i-1}\right|^{\frac{4}{3}-\frac{d}{4}} \\
& \times \cdots \times\left|t-s_{1}\right|^{\frac{4}{3}-\frac{d}{4}} d s_{i} \cdots d s_{1} \\
= & \text { const. } \quad \int_{0}^{t} \int_{0}^{s_{1}} \cdots \int_{0}^{s_{i-2}} \frac{s_{i-1}^{\left(\frac{4}{3}-\frac{d}{4}+1\right)}}{\frac{4}{3}-\frac{d}{4}+1} \\
& \times\left|s_{i-2}-s_{i-1}\right|^{\frac{4}{3}-\frac{d}{4}} \times \cdots \times\left|t-s_{1}\right|^{\frac{4}{3}-\frac{d}{4}} d s_{i-1} \cdots d s_{1} .
\end{aligned}
$$

By a similar argument as in the proof of Theorem 2.1, we get

$$
\begin{aligned}
\widetilde{I}_{i}(t, x) & \leq \text { const. } \quad t^{i\left(1+\frac{4}{3}-\frac{d}{4}\right)} \prod_{k=1}^{i-1} \int_{0}^{1} a^{k\left(\frac{7}{3}-\frac{d}{4}\right)}(1-a)^{\frac{4}{3}-\frac{d}{4}} d a \\
& \leq \text { const. } \quad t^{i\left(1+\frac{4}{3}-\frac{d}{4}\right)} \prod_{k=1}^{i-1} \frac{1}{k\left(\frac{7}{3}-\frac{d}{4}\right)+1} \\
& \leq \text { const. } \quad \frac{t^{i\left(1+\left(\frac{4}{3}-\frac{d}{4}\right)\right)}}{\left(\frac{7}{3}-\frac{d}{4}\right)^{i-1}} \times \frac{1}{(i-1) !}
\end{aligned}
$$


By the boundness of $u_{0}$, for $t>0$ large enough,

$$
\begin{aligned}
E\left[|u(t, x)|^{2}\right] & \leq \text { const. } t^{1+\left(\frac{4}{3}-\frac{d}{4}\right)} \sum_{i=1}^{\infty}\left[\frac{t^{1+\left(\frac{4}{3}-\frac{d}{4}\right)}}{\frac{7}{3}-\frac{d}{4}}\right]^{i-1} \times \frac{1}{(i-1) !} \\
& =\text { const. } t^{1+\left(\frac{4}{3}-\frac{d}{4}\right)} \exp \left[\frac{t^{1+\left(\frac{4}{3}-\frac{d}{4}\right)}}{\frac{7}{3}-\frac{d}{4}}\right]
\end{aligned}
$$

which implies

$$
\limsup _{t \rightarrow \infty} \frac{\log \left\{\sup _{x \in \mathbf{D}} E\left[|u(t, x)|^{2}\right]\right\}}{t^{1+\left(\frac{4}{3}-\frac{d}{4}\right)}} \leq \frac{1}{\frac{7}{3}-\frac{d}{4}} .
$$

This proves the theorem.

Finally, by a similar way as in Section 2, we also get the convergence estimate for the series solution of Equation (1.4) when $d=4,5$.

Proposition 3.1. Let $d=4,5$ and $N \in \mathbf{N}$. Define the first $N$-finite chaos terms of the solution of (1.4) by

$$
\widetilde{u}_{N}(t, x)=\sum_{i=0}^{N} \widetilde{H}_{i}(t, x) .
$$

Then for any $T>0$, there exists a constant $\widetilde{c}>0$ such that, for all $(t, x) \in[0, T] \times \mathbf{D}$,

$$
E\left[\left|u(t, x)-\widetilde{u}_{N}(t, x)\right|^{2}\right] \leq \widetilde{c} b(d, N),
$$

with

$$
b(d, N):=\frac{T^{1+\left(\frac{4}{3}-\frac{d}{4}\right)}}{N !}\left[\frac{T^{1+\left(\frac{4}{3}-\frac{d}{4}\right)}}{\frac{7}{3}-\frac{d}{4}}\right]^{N}
$$

and $\lim _{N \rightarrow \infty} b(d, N)=0$.

\section{ACKNOWLEDGEMENT}

The authors would like to thank their supervisor, Professor Yongjin Wang at Nankai University, for his careful reading of the first version of the manuscript and for making many detailed revisions as well.

\section{REFERENCES}

[1] L. Bo, Y. Wang, Stochastic Cahn-Hilliard partial differential equations with Lévy spacetime noises, Stoch. Dyn. 6 (2006), 229-244. MR2239091 (2007i:60074)

[2] L. Bo, Y. Wang, and L. Yan, Higher-order Stochastic partial differential equations with branching noises, Front. Math. China 3 (2008), 15-35. MR2373092

[3] C. Cardon-Weber, Cahn-Hilliard stochastic equation: existence of the solution and of its density, Bernoulli 7 (2001), 777-816. MR1867082(2002i:60109)

[4] C. Cardon-Weber and A. Millet, On strongly Petrovskin's parabolic SPDEs in arbitrary dimension and application to the stochastic Cahn-Hilliard equation, J. Theory Prob. 17 (2004), 1-49. MR2054575 (2005f:60136)

[5] R. Dalang, Extending the martingale measure stochastic integral with applications to spatially homogeneous SPDEs, Electron. J. Prob. 4 (1999), 1-29. MR1684157(2000b:60132)

[6] Y. Hu, Chaos expansion of heat equations with white noise potentials, Potential Anal. 16 (2002), 45-66. MR 1880347 (2002k:60133)

[7] C. Mueller, Long time existence for the heat equation with a noise term, Prob. Th. Rel. Fields 90 (1991), 505-517. MR1135557 (93e:60120) 
[8] C. Mueller and R. Tribe, A measure-valued process related to the parabolic Anderson model, Progress in Prob. 52, Birkhäuser, Basel (2002), 219-227. MR 1958819

[9] D. Nualart and B. Rozovskii, Weighted stochastic Sobolev spaces and bilinear SPDEs driven by space-time white noise, J. Funct. Anal. 149 (1997), 200-225. MR:1471105 (98m:60100)

[10] D. Nualart and M. Zakai, Generalized Brownian functionals and the solution to a stochastic partial differential equation, J. Funct. Anal. 84 (1989), 279-296. MR1001461 (90m:60076)

[11] H. Uemura, Construction of the solution of 1-dimensional heat equation with white noise potential and its asymptotic behavior, Stoch. Anal. Appl. 14 (1996), 487-506. MR1402691 (97e:60109)

[12] J. Walsh, An introduction to stochastic partial differential equations, Lecture Notes in Math. 1180, Springer, Berlin (1986), 265-439. MR876085 (88a:60114)

School of Mathematical Sciences, Nankai University, Tianjin 300071, People's Republic of China

E-mail address: dantangcn@yahoo.com.cn

Department of Mathematics, Xidian University, Xi'an 710071, People's Republic of CHINA

E-mail address: bolijunnk@yahoo.com.cn 